Check for updates

Cite this: Nanoscale Adv., 2019, 1, 4082

Received 11th June 2019

Accepted 8th September 2019

DOI: 10.1039/c9na00368a

rsc.li/nanoscale-advances

\section{Atomic layer deposition of palladium nanoparticles on a functional electrospun poly-cyclodextrin nanoweb as a flexible and reusable heterogeneous nanocatalyst for the reduction of nitroaromatic compounds†}

\begin{abstract}
Fuat Topuz (iD) *a and Tamer Uyar (D)*ab
We here show a rational approach for the fabrication of a flexible, insoluble catalytic electrospun nanoweb of cross-linked cyclodextrin (CD) for the reduction of nitroaromatics. CD nanofibers were produced by electrospinning an aqueous HP- $\beta-C D$ solution containing a multifunctional cross-linker (i.e., 1,2,3,4butanetetracarboxylic acid, BTCA) and were subsequently cross-linked by heat treatment, which led to an insoluble electrospun poly- $C D$ nanoweb. The poly- $C D$ nanoweb was decorated with $\mathrm{Pd}$ nanoparticles (Pd-NPs) by atomic layer deposition (ALD) technique over 20 cycles to give rise to a catalytic electrospun nanoweb (i.e., Pdapoly-CD). The formation of the Pd-NPs on the poly-CD nanofiber surface was clearly evidenced by TEM and STEM imaging, which displayed the homogeneously distributed Pd-NPs with a mean size of $4.34 \mathrm{~nm}$. ICP-MS analysis revealed that the Pd content on the Pdapoly-CD nanoweb was $0.039 \mathrm{mg}$ per $\mathrm{mg}$ of nanoweb. The catalytic performance of the PdapolyCD nanoweb was tested for the reduction of a nitroaromatic compound (i.e., 4-nitrophenol (4-NP)), and high catalytic performance of the Pdapoly-CD nanoweb was observed with a corresponding TOF value of $0.0316 \mathrm{~min}^{-1}$. XPS was used to explore the oxidation state of Pd atoms before and after the catalytic reduction of 4-NP, and no significant change was observed after catalytic reactions. In brief, the Pdapoly-CD nanoweb having handy, flexible, structural stability and reusability can be effectively used in environmental applications as a heterogeneous nanocatalyst for the reduction of toxic nitroaromatics.
\end{abstract}

\section{Introduction}

Electrospun nanowebs have sparked great interest owing to their high aspect ratio and tailor-made properties for many different applications, including water treatment, ${ }^{\mathbf{1}}$ drug delivery, ${ }^{2}$ wound dressing, ${ }^{3}$ tissue engineering, ${ }^{4}$ sensing, ${ }^{5}$ food ${ }^{6}$ and food packaging, ${ }^{7}$ and catalysis. ${ }^{8}$ Particularly, the use of electrospun nanowebs as supporting materials in heterogeneous catalysis has gained interest recently due to the facile preparation of such nanofibrous catalysts with high catalytic activity. In this regard, various approaches have been developed to produce catalytic nanowebs, such as (i) the electrospinning of polymer solutions with the impregnated metal precursors and thereafter, thermal or chemical treatment of the nanofibers, ${ }^{9}$ (ii)

${ }^{a}$ Institute of Materials Science \& Nanotechnology, Bilkent University, Ankara 06800, Turkey.E-mail: fuat.topuz@rwth-aachen.de

${ }^{b}$ Department of Fiber Science \& Apparel Design, College of Human Ecology, Cornell University, Ithaca, NY 14853, USA. E-mail: tu46@cornell.edu

$\dagger$ Electronic supplementary information (ESI) available: A video showing the handy flexible structure of the Pd@poly-CD nanoweb and the experimental details and results regarding MB sorption. See DOI: 10.1039/c9na00368a in situ synthesis of catalytic particles in nanofibers using reducing agents, ${ }^{10}$ and (iii) the decoration of colloidal metal nanoparticles onto the fiber surface. ${ }^{11}$ In addition to these approaches, atomic layer deposition (ALD) of metals or metal oxides onto electrospun nanowebs has emerged as a more advantageous technique since it promotes the growth of nanoparticles in an atomically controlled manner by chemical attachment to surface oxygen atoms and therefore, allows producing nanoparticles or ultrathin nanolayers on the supporting materials. ${ }^{12-18}$ As the entire process follows a layer-bylayer growth, the deposition of atoms gives rise to nanostructures with atomically controlled interfaces. Furthermore, ALD as a chemical vapor deposition technique enables the chemical attachment of atoms and their subsequent growth into nanoparticles or nanolayers so that the materials can be reused many times without the loss or with a minimal loss of catalytic nanomaterials from the material's surface. ${ }^{\mathbf{1 4}}$ The embedding of nanoparticles in the fiber matrix may reduce their catalytic performance due to accessibility problems of reactants to the nanoparticle interface, and hence, the surface functionalization of electrospun nanowebs with catalytic nanoparticles via ALD has considerable advantages owing to the 
highly accessible surface functionality and the lack of interference from reducing agents or other molecules. In this regard, our research group has made a significant contribution in this area by exploiting different combinations of electrospun nanofibrous materials as substrates and ALD of metal oxides and metals for catalytic purposes. ${ }^{12-16,19}$

The ALD technique is based on self-terminating gas-solid reactions ${ }^{\mathbf{2 0}}$ and is mainly composed of repetitive cycles of the following reactions: (i) the reaction of a metal-ligand (ML) molecule and (ii) the reaction of water. ${ }^{21}$ The first step is likely to happen through ligand (L) swap with one or more surface $\mathrm{OH}$ groups, releasing a gaseous product, HL, while attaching the metal-ligand (ML) species to the surface. Thereafter, water molecules react with the adsorbed ligands and replace them with $\mathrm{OH}$ groups by releasing more HL. In the following cycle, the hydroxyl groups react again with the ML molecules. Hence, there is a correlation between the growth-per-cycle and the number of surface hydroxyl groups. ${ }^{21}$ In this regard, the use of an electrospun poly-cyclodextrin (poly-CD) nanoweb as a supporting material facilitates the initial deposition of metal atoms owing to the presence of many hydroxyl groups on the molecular structure of CD. Subsequently, cyclic repetition produces nanoparticles or ultrathin nanolayers on the nanofibers depending on the applied ALD cycle number. ${ }^{\mathbf{1 3}}$

Because of their high catalytic activity and intrinsic longterm stability against corrosion, Pd nanoparticles (Pd-NPs) have been exploited as heterogeneous catalysts for many reactions, including Suzuki-Miyaura coupling, ${ }^{22,23}$ Heck, ${ }^{24-26}$ Stille coupling, ${ }^{27,28}$ Sonogashira ${ }^{29,30}$ and nitroarene reduction. ${ }^{31}$ Several approaches have been exploited for the chemical decoration of the fiber surface with Pd-NPs and other nanoparticles to obtain handy, flexible catalytic materials with the further advantage of reusability. ${ }^{15,19,32}$ In this regard, the use of Pd-NPs with ALD enables the preparation of highly catalytic materials, and the deposition of Pd atoms takes place as a layer-by-layer process so that the layer growth can be achieved with fine control. ${ }^{15}$ Such electrospun nanowebs can be exploited for the catalytic conversion of hazardous water pollutants to less harmful ones, such as nitroaromatics. ${ }^{15}$

Nitroaromatics, i.e., nitro-substituted aromatic hydrocarbons, are exploited in the synthesis of key intermediates for pharmaceuticals $^{33,34}$ and agrochemicals. ${ }^{35}$ Most of them are toxic and labeled as carcinogenic molecules due to their adverse effects on human health. Among them, 4-nitrophenol (4-NP) has been used for different applications, including as the substrate for glucuronide and sulfate conjugation pathways, ${ }^{36}$ and the synthesis of organophosphorus insecticide derivatives. $^{37}$ However, 4-NP is a very poisonous and refractory pollutant, which is hence incorporated in the US Environmental Protection Agency (USEPA) "Priority Pollutant List". ${ }^{38}$ The degradation or transformation of such toxic chemicals into less harmful derivatives is highly suggested to mitigate their toxicity. In this regard, various catalytic approaches have been applied for the transformation of nitro-groups into less harmful derivatives. ${ }^{39}$ Recently, polymer-free cyclodextrin electrospun nanofibers with in situ formed Pd nanoparticles were produced and used for the reduction of $4-\mathrm{NP} .{ }^{10}$ On contact with water, the embedded Pd-NPs are released from the fiber matrix and efficiently catalyze the reduction of 4-NP. However, such purely cyclodextrin-based nanofibers suffer from non-reusability because of their instant dissolution in aqueous solutions.

Electrospun nanofibers having the cyclodextrin (CD) functionality are quite interesting since $\mathrm{CD}$ molecules can form inclusion complexes with water pollutants. The use of CD-based materials as the supporting materials for the deposition of $\mathrm{Pd}$ nanoparticles allows their use as an adsorbent besides their application as a nanocatalyst since functional CD molecules can sequester micropollutants from water through inclusioncomplexation. In this regard, recently we reported the fabrication of poly-CD nanowebs using tetracarboxylic acid ${ }^{\mathbf{4 0 , 4 1}}$ or epichlorohydrin ${ }^{42}$ based cross-linkers. Both cross-linking routes led to structurally stable and insoluble nanofibrous poly-CD membranes, which could efficiently scavenge water pollutants.

In this study, an electrospun poly-CD nanoweb was produced, and the nanofiber surface was functionalized with

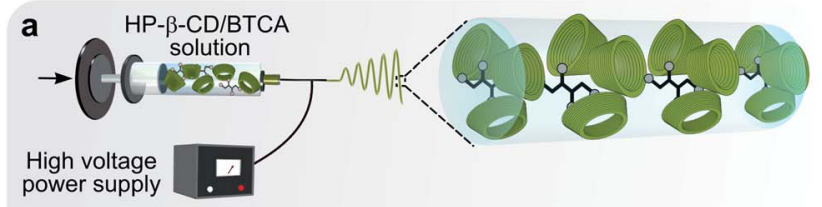

Production of the poly-CD electrospun nanoweb
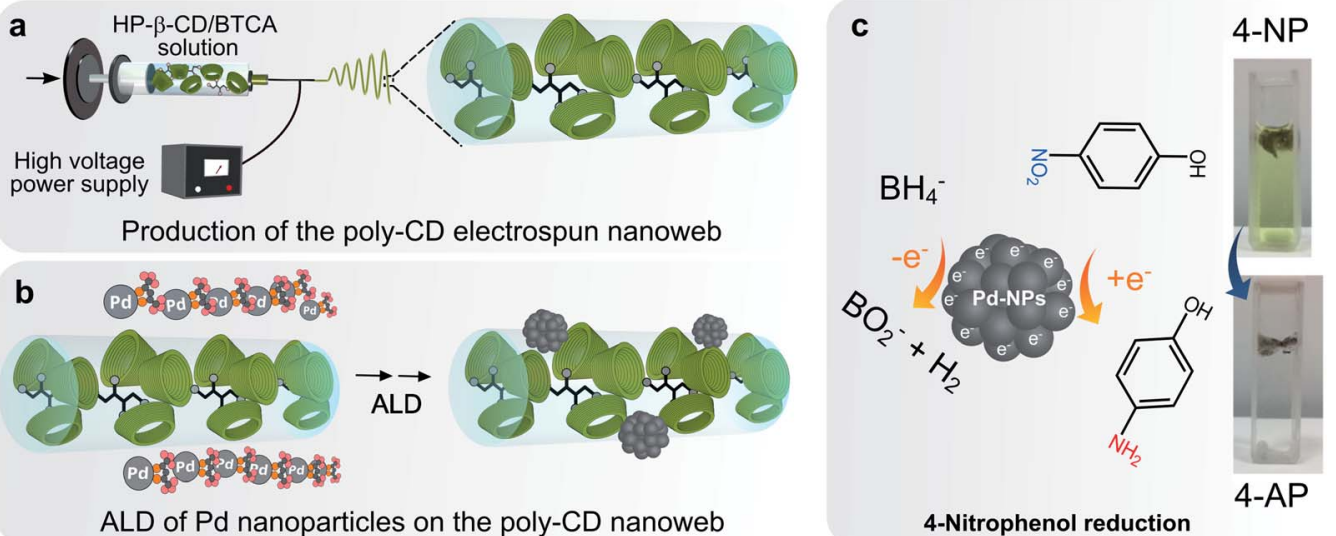

Fig. 1 (a) A schematic representation of the production of an electrospun poly-CD nanoweb and (b) subsequent surface decoration of the nanoweb with Pd-NPs by ALD. A cartoon illustration of the use of the Pdapoly-CD nanoweb in (c) the catalytic reduction of 4-NP. Photos show the 4-NP solution before and after treatment with the Pdapoly-CD nanoweb. 
Pd-NPs by ALD. The nanoweb was exploited for the reduction of a nitroaromatic compound, 4-NP, in water (Fig. 1). The poly-CD nanoweb was prepared by electrospinning an aqueous solution of HP- $\beta$-CD containing tetracarboxylic acid functional linkers, followed by their cross-linking with heat treatment. The nanoweb was used as the supporting material for ALD to grow Pd atoms in the form of nanoparticles on the nanofiber surface, and the resultant material was characterized in terms of morphology by SEM and TEM, and compositionally by XPS, ICPMS, and EDS. The catalytic activity of the Pd-decorated poly-CD nanoweb was tested against the reduction of 4-NP.

\section{Experimental}

\section{Materials}

Hydroxypropyl- $\beta$-cyclodextrin (HP- $\beta$-CD) was received as a gift sample from Wacker Chemie (Germany). 1,2,3,4-Butanetetracarboxylic acid (BTCA) (99\%, Sigma Aldrich) and sodium hypophosphite hydrate (SHP, $\geq 99 \%$, Sigma Aldrich) were obtained. 4-Nitrophenol (4-NP, 99\%, Alfa Aesar), sodium borohydride $\left(\mathrm{NaBH}_{4}, 98 \%\right.$, fine granular, Merck), methanol $(\mathrm{MeOH}$, Sigma-Aldrich), and methylene blue (MB, Sigma Aldrich) were used as received. Palladium(II) hexafluoroacetylacetonate $\left(\operatorname{Pd}(\text { hfac })_{2}\right)$ was purchased from Strem Chemicals.

\section{Production of the electrospun poly-CD nanoweb}

First, HP- $\beta$-CD $(155 \%$, w/v) was dissolved in water, and the solution was stirred at $25{ }^{\circ} \mathrm{C}$ until a clear solution was obtained. BTCA (20 wt\% with respect to the HP- $\beta$-CD) and SHP ( $2 \mathrm{wt} \%$ with respect to the HP- $\beta$-CD) were added to the solution. The mixture was stirred at $50{ }^{\circ} \mathrm{C}$ under continuous stirring, and thereafter, it was cooled down to RT. For electrospinning, the solution was transferred into a plastic syringe connected with a needle (inner diameter: $0.4 \mathrm{~mm}$ ). The solution was pushed through a syringe pump (Model: KDS-101, KD Scientific) at a feeding rate of $1 \mathrm{~mL} \mathrm{~h}^{-1}$. A piece of aluminum foil was used to cover a grounded metal collector, which was placed at a $10 \mathrm{~cm}$ distance from the needle head. A high voltage power supply (AU Series, Matsusada Precision Inc.) was used to provide a fixed electrical potential of $10 \mathrm{kV}$. Finally, the nanofibers were thermally treated in an oven at $175^{\circ} \mathrm{C}$ for 1 hour to produce a crosslinked, insoluble electrospun poly-CD nanoweb.

\section{ALD of Pd nanoparticles on the electrospun poly-CD nanoweb}

ALD was exploited on the electrospun poly-CD nanoweb for the deposition of Pd-NPs on the nanofiber surface using a Pd precursor $\left(\mathrm{Pd}(\mathrm{hfac})_{2}\right)$ and a counter reactant, formalin $(\mathrm{HCOH}$, $30 \%$ in aqueous solution with $15 \%$ methanol). $\mathrm{Pd}(\text { hfac })_{2}$ was kept at $70{ }^{\circ} \mathrm{C}$ to obtain a suitable vapor pressure. A carrier gas $\left(\mathrm{N}_{2}\right)$ with a flow rate of $20 \mathrm{sccm}$ was used under dynamic vacuum conditions. Twenty cycles were applied for the deposition of Pd on the nanofibers at $200{ }^{\circ} \mathrm{C}$. The exposure mode conditions for the formalin and Pd were as follows: $0.1 \mathrm{~s}$ pulse, 2 min holding time and $1 \mathrm{~s}$ pulse, and 2 min holding time.

\section{Characterization}

The morphology of the Pd@poly-CD nanoweb was analyzed by scanning electron microscopy (SEM, Quanta FEI 200). Before SEM analysis, the nanofibers (i.e., HP- $\beta-C D$, poly-CD, and Pd@poly-CD) were coated with a $10 \mathrm{~nm}$ Au layer using a GATAN PECS $^{\text {TM }}$ sputter coating system. The mean diameter $(\langle D\rangle)$ of the nanofibers was evaluated over 100 nanofibers using ImageJ software (US National Institutes of Health, Bethesda, MD, USA). Transmission electron microscopy (TEM) images were obtained on a Tecnai G2F30 (FEI) apparatus. The nanofiber sample was sonicated in ethanol, and a tiny droplet was dropped and dried on a carbon-coated copper grid. TEM was operated with an accelerating voltage of $300 \mathrm{kV}$. The elemental composition of the electrospun nanoweb was explored by energy-dispersive $\mathrm{X}$ ray spectroscopy (EDS). Scanning transmission electron microscopy (STEM) mode was used to observe the Pd-NPs on the nanofibers. The X-ray photoelectron spectra of the samples were recorded using an X-ray photoelectron spectrometer (Thermo Fisher Scientific, U.K.). As an X-ray source, an Al Kalpha X-ray monochromator (0.1 eV step size, $12 \mathrm{kV}, 2.5 \mathrm{~mA}$, with a spot size of $400 \mu \mathrm{m}$ ) was exploited at an electron take-off angle of $90^{\circ}$. The deconvoluted XPS spectrum of the Pd $3 \mathrm{~d}$ orbital was measured 30 times with $50 \mathrm{~ms}$ dwell time (pass energy $30 \mathrm{eV}$ ). A PANalytical X'Pert Pro MPD was used to obtain XRD patterns from the samples. The equipment has a Philips PW3040/60 X-ray generator and fitted with an X'celerator detector. The sample was exposed to $\mathrm{Cu} \mathrm{K}$-alpha X-ray radiation $(\lambda=1.541874 \AA)$ to obtain diffraction data. The X-rays were produced from a $\mathrm{Cu}$ anode that was supplied with $40 \mathrm{kV}$ and a current of $40 \mathrm{~mA}$. The data were obtained from a $2 \theta$ range of $30-90^{\circ}$. The Pd content on the nanoweb was determined by inductively coupled plasma-mass spectroscopy (ICP-MS, Thermo $\mathrm{X}$ series-II inductively coupled plasma-mass spectrometer). In this regard, the Pd@poly-CD nanoweb was treated with aqua regia ( $2 \mathrm{mg}$ nanoweb in $2 \mathrm{~mL}$ of aqua regia). From this solution, $100 \mu \mathrm{L}$ was taken, and the volume was made up to $10 \mathrm{~mL}$ with $2 \% \mathrm{HNO}_{3}$.

\section{Catalytic experiments}

An aqueous 4-NP solution ( $2.9 \mathrm{~mL}, 0.2 \mathrm{mM}$ ) was transferred into a cuvette. Subsequently, $100 \mu \mathrm{L}$ of $0.3 \mathrm{M} \mathrm{NaBH}_{4}$ was added. After the addition of the Pd@poly-CD nanoweb ( $2 \mathrm{mg}$ ), UV-vis spectra were collected on a Cary-UV 100 spectrophotometer every $5 \mathrm{~min}$ in the wavelength range of 250-500 $\mathrm{nm}$. The peak intensity at $400 \mathrm{~nm}$ was exploited for the conversion of 4-NP to 4-AP. The measurements were conducted at $25^{\circ} \mathrm{C}$. The same nanoweb was reused four times using identical concentrations of 4-NP and $\mathrm{NaBH}_{4}$.

\section{Results and discussion}

Production and characterization of the electrospun Pd@polyCD nanoweb

The HP- $\beta$-CD nanofibers were produced by electrospinning an aqueous HP- $\beta$-CD solution $(c=155 \%(\mathrm{w} / \mathrm{v}))$ containing a crosslinker, BTCA, and a catalyst, SHP. Such a cross-linking reaction 

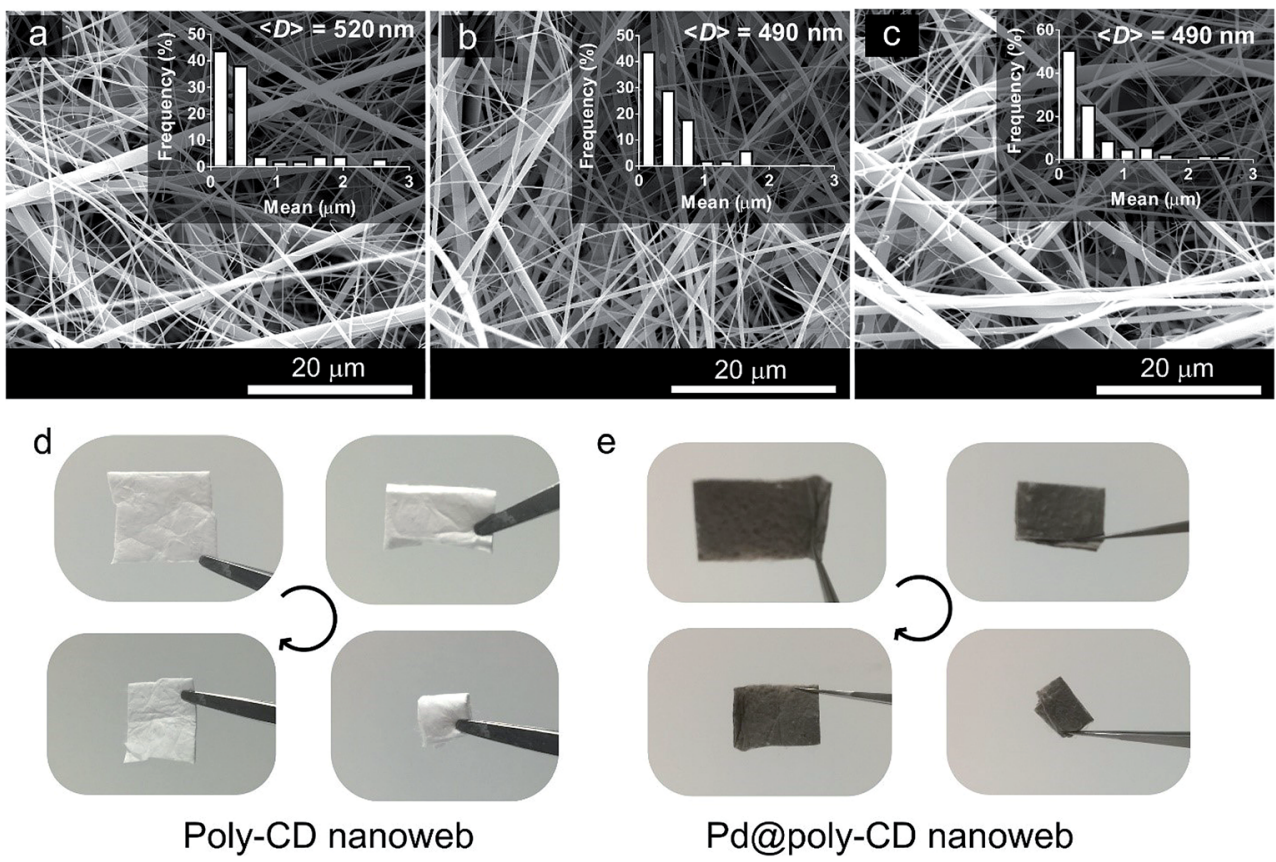

Fig. 2 Scanning electron micrographs of the electrospun (a) HP- $\beta-C D$, (b) poly-CD and (c) Pdapoly-CD nanofibers. Insets display the sizedistributions of the nanofibers. (d) Optical photos of the poly-CD nanoweb (d) before and (e) after the decoration with Pd-NPs by ALD.

between carboxylic acid and CD was adapted from Martel et al. $^{\mathbf{4 3}}$ After the electrospinning process, the nanofibers in various sizes were produced with a mean fiber diameter of $520 \mathrm{~nm}$ (Fig. 2a). Afterward, the nanofibrous web was heat-treated in an oven at $175^{\circ} \mathrm{C}$ for $1 \mathrm{~h}$ to generate an insoluble electrospun polyCD nanoweb. The SEM image of nanofibers in the poly-CD nanoweb shows the bead-free nanofiber structure with a mean fiber diameter of $490 \mathrm{~nm}$ (Fig. 2b), demonstrating that the crosslinking process does not cause any significant change in the fiber size. The poly-CD nanofibers were decorated with Pd-NPs by the ALD technique. During the ALD process, 20 cycles were applied to ensure the deposition of Pd in the form of nanoparticles (Pd-NPs) on the fiber surface, rather than the formation of a thin film of Pd since the high accessibility of Pd atoms is crucial for the enhanced catalytic performance of the poly-CD nanoweb. As we previously obtained small Pd-NPs on polyamide and polyacrylonitrile fibers by performing ALD over 15 cycles, ${ }^{15}$ we increased the cycle number to 20 to produce catalytic nanofibers with densely coated Pd-NPs. The SEM image of the Pd decorated poly-CD nanofibers did not reveal any structural changes on the fibers, and the mean fiber diameter remained unchanged (i.e., $490 \mathrm{~nm}$ ) (Fig. 2c). After the ALD step, the white color of the poly-CD nanoweb turned into dark grey, suggesting the successful deposition of Pd atoms onto the poly-CD nanoweb (Pd@poly-CD nanoweb) (Fig. 2d and e). Furthermore, the resultant freestanding $\mathrm{Pd} @$ poly-CD nanoweb could be folded without any breaking, demonstrating its flexible character under multiple folding (ESI Video $1 \dagger$ ).

The deposition of the Pd-NPs on the poly-CD nanoweb was confirmed by TEM and STEM, which revealed homogeneously distributed Pd-NPs on the Pd@poly-CD nanofibers (Fig. 3a and b). The average size of the Pd-NPs was measured as $4.34 \mathrm{~nm}$, showing the formation of small Pd-NPs due to low ALD cycle number. ${ }^{44,45}$ The crystalline structure of the Pd-NPs was explored using the selected area electron diffraction (SAED) pattern. The SAED pattern of the nanofibers revealed diffuse
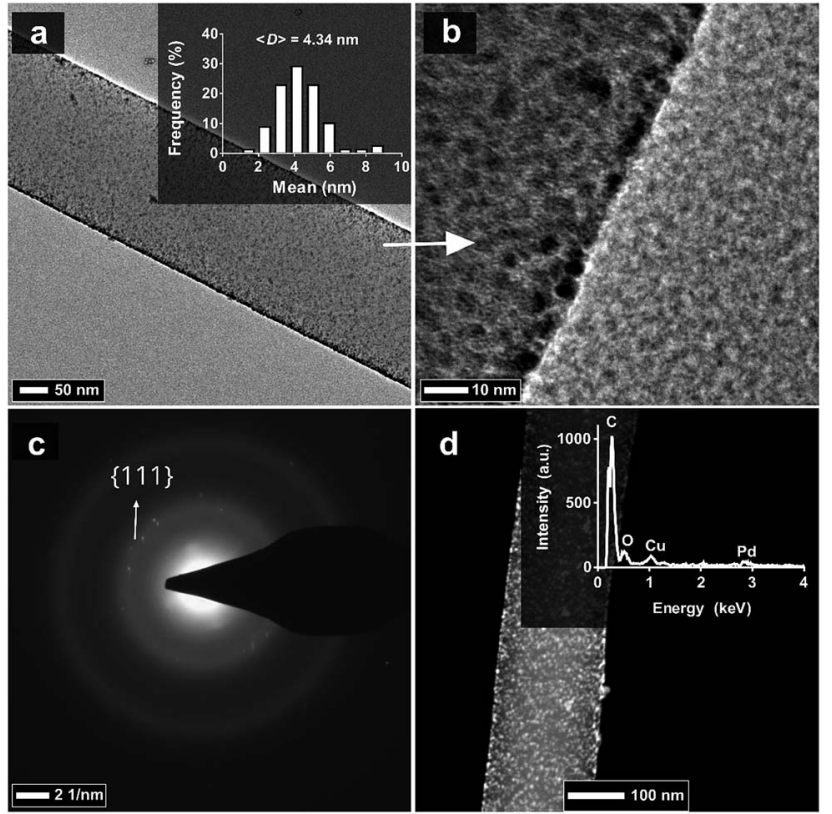

Fig. 3 ( $a$ and b) Transmission electron micrographs of the PdapolyCD nanofibers. The inset shows the plot of particle-size distribution. (c) The SAED pattern of the Pdapoly-CD nanofibers. (d) Scanning transmission electron micrograph of a Pdapoly-CD nanofiber. The inset displays the EDS spectrum of the respective nanofibers. 
rings due to an amorphous poly-CD fiber matrix plus bright diffraction spots from a $\operatorname{Pd}\{111\}$ plane (Fig. 3c). Further, XRD analysis of the pristine poly-CD nanoweb revealed a broad peak at $18.59^{\circ}$ (Fig. S1a, ESI $\dagger$ ). Likewise, the Pd@poly-CD nanoweb showed a broad peak at $18.59^{\circ}$ due to the amorphous structure of the HP- $\beta-C D$, together with a small diffraction peak of the $\{111\}$ plane of face-centered cubic (fcc) Pd at $40^{\circ}(d$-spacing $=$ $2.26 \AA$ ) (Fig. S1 $\mathrm{b}_{\dagger}^{\dagger}$ ), which is in agreement with literature reports. ${ }^{46}$ The absence of other peaks regarding Pd-NPs in the XRD pattern can be attributed to the low concentration of the Pd-NPs on the nanofibers. Likewise, such peaks were not detected by XRD for the ALD-mediated Pd-NP decorated Nylon and polyacrylonitrile nanofibers because of the low weight loading of $\mathrm{Pd} .{ }^{15}$ As the content of the amorphous poly-CD matrix dominates the composition, the presence of the Pd-NPs was not revealed as crystalline peaks, except for the dominant peak of a $\operatorname{Pd}\{111\}$ plane. The STEM image of the Pd@poly-CD nanofibers revealed the homogeneous distribution of the Pd-NPs throughout the longitudinal axis of the nanofibers (Fig. 3d). The inset shows the EDS analysis of the Pd@poly-CD nanoweb, which revealed the presence of Pd-NPs on the Pd@poly-CD nanofiber surface because of the successful deposition of Pd atoms on the nanofibers by ALD. Furthermore, ICP-MS analysis was performed to quantitate the Pd content on the Pd@poly-CD nanofibers, and the presence of $0.039 \mathrm{mg} \mathrm{mg}^{-1} \mathrm{Pd}$ on the Pd@poly-CD nanoweb was determined.

\section{Reduction of 4-nitrophenol by the electrospun Pd@poly-CD nanoweb}

The catalytic activity of the Pd@poly-CD nanoweb was tested in the reduction of a nitroaromatic compound, 4-nitrophenol (4NP). 4-NP exists in the industrial wastewater from the production of herbicides ${ }^{47}$ and dyestuffs. ${ }^{48}$ Nitro groups are easily reduced to give amino compounds. ${ }^{49}$ Such a transformation leads to its less harmful derivative (i.e., 4-aminophenol, 4-AP), which is highly desired to mitigate its toxicity.

The reduction of 4-NP by the poly-CD and Pd@poly-CD nanowebs was explored (Fig. 4). Normally, 4-NP gives a maximum absorption at $316 \mathrm{~nm}$ while upon the addition of $\mathrm{NaBH}_{4}$, phenolate ions form, which shifts the maximum absorption peak to $400 \mathrm{~nm}$. Fig. 4 a shows the changes in the UVvis spectra of 4-NP in the presence of the pristine Pd-free polyCD nanoweb, where a very small decrease in the peak intensity was observed. This can be attributed to the reduction of 4$\mathrm{NP}$ by $\mathrm{NaBH}_{4}$ and the partial adsorption of 4-NP onto the polyCD nanoweb. However, the interference from the CD cavity was insignificant in the presence of the Pd@poly-CD nanoweb, and the use of the Pd@poly-CD nanoweb drastically decreased the
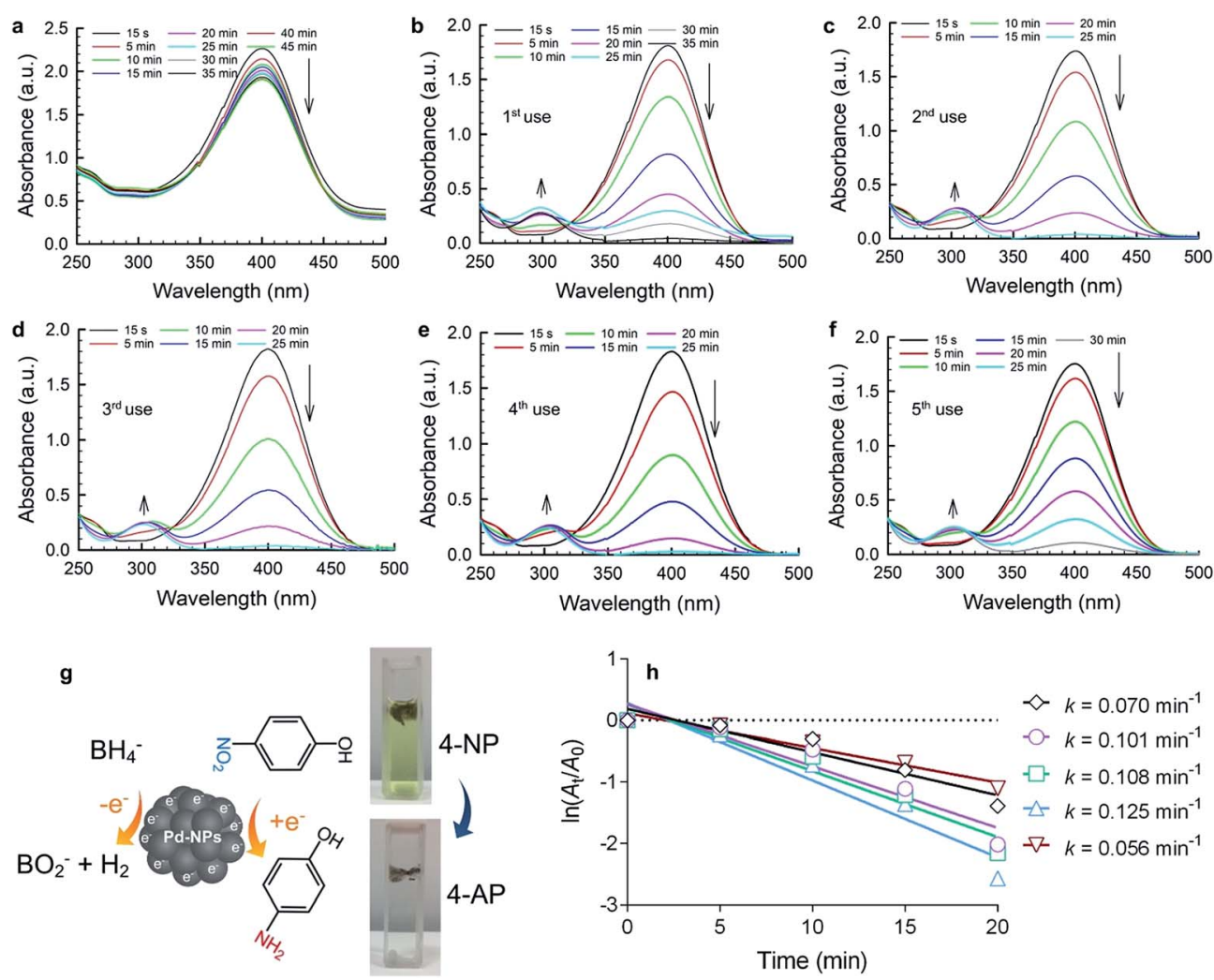

Fig. 4 The catalytic performance of (a) the poly-CD and (b) Pdapoly-CD nanowebs for the reduction of 4-NP to 4-AP, monitored by UV-vis, at RT over time (time interval is $5 \mathrm{~min}$ ). The use of the same nanoweb for (c) $2^{\text {nd }}$, (d) $3^{\text {rd }}$, (e) $4^{\text {th }}$, and (f) $5^{\text {th }}$ times for the reduction of $4-\mathrm{NP}$. (g) The reduction pathway of 4-NP to 4-AP with the respective photos of the solutions before and after reduction. (h) A plot of $\ln \left(A_{t} / A_{0}\right)$ versus time for the reduction of 4-NP catalyzed by the Pdapoly-CD nanoweb during its repetitive use. The measurements were performed at $25{ }^{\circ} \mathrm{C}$. $5.8 \times$ $10^{-4} \mathrm{mmol} 4-\mathrm{NP}$ and $2 \mathrm{mg}$ Pdapoly-CD nanoweb were used for the catalytic experiments. 
absorption peak intensity at $400 \mathrm{~nm}$ because of the reduction of 4-NP by the Pd-NPs. A new peak appeared at $300 \mathrm{~nm}$ due to the formation of 4-aminophenol (4-AP), and an inverse correlation between the two peaks was observed (Fig. 4b). After the complete reduction of $4-\mathrm{NP}$, the nanoweb was reused for the reduction of the identical concentration of 4-NP (Fig. 4c). Similar catalytic performance of the Pd@poly-CD nanoweb was observed. Furthermore, the nanoweb could be reused three times more without any drastic drop in its catalytic performance (Fig. 4d-f). The Pd-mediated reduction reaction of $4-\mathrm{NP}$ is shown in Fig. $4 \mathrm{~g}$, where the $\mathrm{NO}_{2}$ group was reduced to $\mathrm{NH}_{2}$ by the Pd-NPs in the presence of $\mathrm{NaBH}_{4}$, and with that, the color of the respective solution turned from yellowish to colorless, demonstrating the formation of 4-AP. During the reduction experiments, a higher amount of $\mathrm{NaBH}_{4}$ was used to ensure that the reaction follows pseudo-second-order kinetics with respect to the 4-NP only. Thus, the reaction kinetics can be described as $-k t=\ln \left(A_{\mathrm{t}} / A_{0}\right)$, where $k$ is the first-order rate constant, $t$ is the reaction time while $A_{\mathrm{t}}$ and $A_{0}$ are the absorbance values at times $t$ and 0 , respectively. The rate constants $(k)$ were determined from the slopes of the fitted lines (Fig. $4 \mathrm{~h}$ ). The mean reaction constant $k$ was found to be $0.092 \mathrm{~min}^{-1}$, and it remained nearly stable during the reuse of the Pd@poly-CD nanoweb, suggesting the sustained catalytic activity of the Pd@poly-CD nanoweb for its repetitive use (Fig. 4h). The respective TOF value for the reduction of 4-NP was calculated as $0.0316 \mathrm{~min}^{-1}$.

The detachment of Pd ions from the nanoweb during its use was explored by ICP-MS analysis, which revealed less than $1 \%$ release of Pd ions after the $1^{\text {st }}$ use, and the total released Pd content was lower than $5 \%$ after the $5^{\text {th }}$ use of the same nanoweb. We also performed an experiment on the catalytic activity of the aqueous phase after the removal of the nanoweb and observed no significant catalytic activity of the medium in the 4NP reduction (Fig. S2 $\dagger$ ). The presence of strong interactions between Pd and the polymer interface through the ALD process minimized the leaching of Pd ions from the fiber surface. A similar finding was reported by Ranjith et al., who showed that the catalytic activity of Pd-deposited nylon nanofibers is maintained thanks to the ALD process. ${ }^{50}$ The performance of the electrospun Pd@poly-CD nanoweb in the reduction of 4-NP was much higher than that of monometallic Pd and bimetallic Pd/ $\mathrm{Ag}$ decorated nylon nanowebs reported previously, and the respective TOF values of the nanowebs were calculated to be 1.75 fold lower than that of the Pd@poly-CD nanoweb. ${ }^{50}$ During the Pd-mediated reduction of $4-\mathrm{NP}$, the $\operatorname{Pd}(0)$ accepts electrons from the $\mathrm{BH}_{4}{ }^{-}$ions and favors the production of an $\mathrm{H}$ atom from the fractionation of the $\mathrm{B}-\mathrm{H}$ bond (Fig. $4 \mathrm{~g}$ ). Active $\mathrm{H}$ atoms later react with the 4-nitrophenolate ions by hydrogenation. In this regard, the presence of the $\operatorname{Pd}\{111\}$ plane as revealed by SAED and XRD analyses is also worth mentioning, since it is regarded as the highly interactive site for the reduction of 4NP. ${ }^{15}$ The presence of the $\operatorname{Pd}\{111\}$ plane enhanced the catalytic activity of the Pd@poly-CD nanoweb. Furthermore, owing to its highly cross-linked structure, the Pd@poly-CD nanoweb, unlike uncross-linked CD nanofibers, ${ }^{10}$ can be reused multiple times in water and organic solvents for catalytic applications. Furthermore, owing to the presence of functional CD molecules in the nanoweb, the Pd@poly-CD nanoweb was also exploited as an adsorbent for the removal of methylene blue (MB) dye from water. The sorption results revealed that the nanoweb can be used as a high-efficiency sorbent for the removal of MB from water with an equilibrium adsorption capacity of $104.05 \pm$ $6.27 \mathrm{mg} \mathrm{g}^{-1}$ (Fig. S3-S5 and Table S1 $\dagger$ ), which is higher than the performance of many reported adsorbents (Table S2 $\dagger$ ). Moreover, the morphology of the nanofibers could be maintained after use (Fig. S6†). All relevant data regarding MB removal are available in the ESI. $\dagger$

The chemical state of the Pd atoms before and after the repetitive use of the $\mathrm{Pd} @$ poly-CD nanoweb in the 4-NP reduction was explored through XPS (Fig. 5a and b). The appearance of the Pd peak in the XPS analysis of the Pd@poly-CD nanoweb demonstrates the successful deposition of the Pd-NPs on the nanofiber surface. For the electrospun Pd@poly-CD nanoweb, deconvoluted Pd 3d spectra displayed two peaks related to metallic and oxidized Pd states (Fig. 5a). Fig. 5a shows the deconvoluted Pd 3d XPS spectrum after the catalytic use of the Pd@poly-CD nanoweb, where a doublet of a low-energy Pd 3d band and a high-energy $\mathrm{Pd} 3 \mathrm{~d}_{3 / 2}$ band appeared. The respective binding energies (i.e., $335.7 \mathrm{eV}$ for Pd $3 \mathrm{~d}_{5 / 2}$ and $341.6 \mathrm{eV}$ for Pd $\left.3 \mathrm{~d}_{3 / 2}\right)$ fit well with literature reports. ${ }^{51}$ The percentages of $\operatorname{Pd}(0)$ and $\mathrm{Pd}(\mathrm{II})$ were, respectively, calculated as 73.31 and $26.69 \%$, demonstrating the formation of metallic Pd-NPs while the respective values after its $5^{\text {th }}$ use were 72.43 and $27.57 \%$ (Fig. 5b), demonstrating that their repetitive use in the 4-NP reduction did not significantly affect the oxidation state of $\mathrm{Pd}$.

\section{Structural stability of the electrospun Pd@poly-CD nanoweb after its use in catalysis}

The structural stability of electrospun fibers is crucial for their repetitive use in catalysis. The stability of the Pd@poly-CD nanoweb was explored over the fiber morphology by SEM. Fig. 6 shows the morphology of the nanofibers in the nanoweb, where no significant change was observed after their $5^{\text {th }}$ use in the 4-NP reduction (Fig. 6a). The swelling of the nanofibers was low and a $20 \%$ increase was observed in the fiber diameter, and the size analysis of the nanofibers revealed a similar mean fiber diameter $(\sim 600 \mathrm{~nm})$ (Fig. $6 \mathrm{~b})$ after their use in the 4-NP reduction several times. The structural stability of the nanoweb could be credited to the occurrence of highly efficient crosslinking reactions between the $\mathrm{CD}$ and cross-linker BTCA, which led to a highly stable poly-CD nanoweb (Fig. 6c). The
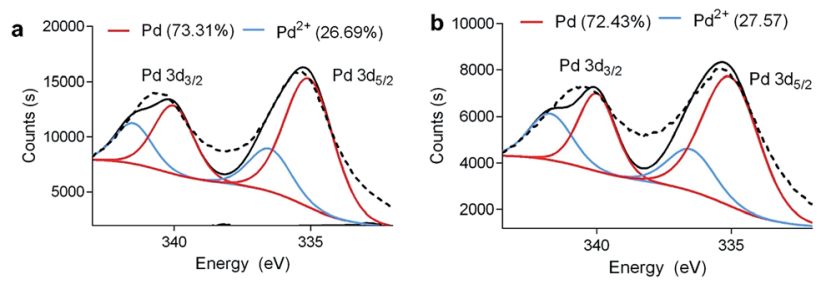

Fig. 5 The deconvoluted Pd 3d XPS spectrum of the Pdapoly-CD nanoweb (a) before and (b) after its repetitive use in the catalytic reduction of 4-NP. 


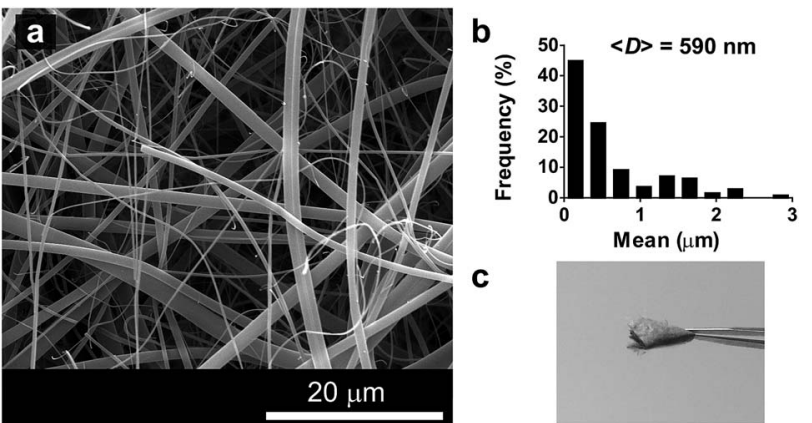

Fig. 6 (a) Scanning electron micrograph of the Pdapoly-CD nanoweb after its 5 th use in the reduction of 4-NP. (b) The size distribution of the respective nanofibers. (c) Optical photo of the dried electrospun mat after its use in the 4-NP reduction.

highly cross-linked structure of the nanoweb and the presence of chemically attached Pd nanoparticles make this nanofibrous material superior to many other catalytic electrospun nanowebs, which are mostly based on hydrophobic uncross-linked polymers $^{52}$ and can dissolve upon exposure to organic media.

\section{Conclusions}

The electrospun poly-CD nanoweb was produced by electrospinning and subsequently functionalizing with ALD of Pd-NPs. The resultant Pd@poly-CD nanoweb was exploited for the catalytic conversion of a nitroaromatic pollutant (4-NP). The bead-free fiber structure of the nanofibers was revealed by SEM analysis, while TEM analysis displayed the homogeneous distribution of ultrasmall Pd-NPs with a mean size of $4.34 \mathrm{~nm}$ on the Pd@poly-CD nanofiber surface. ICP-MS showed the presence of $39 \mu \mathrm{g}$ Pd per mg of the Pd@poly-CD nanoweb sample. XPS measurements confirmed the reduction of $\mathrm{Pd}(\mathrm{II})$ to metallic $\operatorname{Pd}(0)$ to a large extent $(\operatorname{Pd}(0)=73.3 \%)$ while no significant change was revealed in the oxidation state of Pd after its repetitive use, suggesting its sustained catalytic activity. The catalytic performance of the nanofibers was analyzed through the hydrogenation of a nitroaromatic compound (i.e., 4-NP). The results revealed their high catalytic activity in the nitroaromatic reduction, and the respective TOF value for the 4-NP reduction was calculated as $0.0316 \mathrm{~min}^{-1}$. Furthermore, the Pd@poly-CD nanoweb with a high functional CD content acted as an efficient adsorbent for MB removal from an aqueous solution with a sorption capacity of $104.05 \pm 6.27 \mathrm{mg} \mathrm{g}^{-1}$, while the sorption kinetics was found to fit well the pseudo-secondorder model. Due to the structural flexibility, cross-linked structure, and high catalytic activity, the electrospun Pd@poly-CD nanoweb can be a promising material for water treatment applications for the reduction of environmentally hazardous nitroaromatics.

\section{Conflicts of interest}

There are no conflicts to declare.

\section{Acknowledgements}

The authors thank Dr Bushan Patil and Zehra Irem Yildiz for their help with ALD and for producing the poly-CD nanoweb, respectively.

\section{Notes and references}

1 M. L. Focarete, C. Gualandi and S. Ramakrishna, Filtering media by electrospinning: Next generation membranes for separation applications, Springer, 2018.

2 F. Topuz and T. Uyar, Pharmaceutics, 2019, 11(1), 6.

3 S. P. Miguel, R. S. Sequeira, A. F. Moreira, C. S. D. Cabral, A. G. Mendonça, P. Ferreira and I. J. Correia, Eur. J. Pharm. Biopharm., 2019, 139, 1-22.

4 Electrospun Materials for Tissue Engineering and Biomedical Applications: Research, Design and Commercialization, ed, T. Uyar and E. Kny, Woodhead Publishing, 2017.

5 E. Z. Antonella Macagnano and E. Kny, Electrospinning for High Performance Sensing, Springer, 2015.

6 A. Celebioglu, F. Kayaci-Senirmak, S. Ipek, E. Durgun and T. Uyar, Food Funct., 2016, 7, 3141-3153.

7 S. Torres-Giner, in Multifunctional and Nanoreinforced Polymers for Food Packaging, ed. J.-M. Lagarón, Woodhead Publishing, 2011, pp. 108-125, DOI: 10.1533/ 9780857092786.1.108.

8 J. Xue, T. Wu, Y. Dai and Y. Xia, Chem. Rev., 2019, 119, 52985415.

9 A. C. Patel, S. Li, C. Wang, W. Zhang and Y. Wei, Chem. Mater., 2007, 19, 1231-1238.

10 A. Celebioglu, F. Topuz and T. Uyar, New J. Chem., 2019, 43, 3146-3152.

11 K. Karakas, A. Celebioglu, M. Celebi, T. Uyar and M. Zahmakiran, Appl. Catal., B, 2017, 203, 549-562.

12 F. Kayaci, C. Ozgit-Akgun, I. Donmez, N. Biyikli and T. Uyar, ACS Appl. Mater. Interfaces, 2012, 4, 6185-6194.

13 F. Kayaci, C. Ozgit-Akgun, N. Biyikli and T. Uyar, RSC Adv., 2013, 3, 6817-6820.

14 A. Celebioglu, K. S. Ranjith, H. Eren, N. Biyikli and T. Uyar, Sci. Rep., 2017, 7, 13401.

15 K. S. Ranjith, A. Celebioglu, H. Eren, N. Biyikli and T. Uyar, Adv. Mater. Interfaces, 2017, 4, 1700640.

16 M. A. Khalily, M. Yurderi, A. Haider, A. Bulut, B. Patil, M. Zahmakiran and T. Uyar, ACS Appl. Mater. Interfaces, 2018, 10, 26162-26169.

17 G. Zhao, Y. Zhang, L. Zhang, Z. G. Ye, W. Ren, F. Xu, S. Wang, M. Liu and X. Zhang, ACS Biomater. Sci. Eng., 2017, 3, 29002906.

18 J. W. Jung, D. W. Choi, C. K. Lee, K. R. Yoon, S. Yu, J. Y. Cheong, C. Kim, S. H. Cho, J. S. Park, Y. J. Park and I. D. Kim, Nano Energy, 2018, 46, 193-202.

19 O. Arslan, F. Topuz, H. Eren, N. Biyikli and T. Uyar, New J. Chem., 2017, 41, 4145-4156.

20 M. Ritala and M. Leskelä, in Handbook of Thin Films, ed. H. Singh Nalwa, Academic Press, Burlington, 2002, pp. 103159, DOI: 10.1016/b978-012512908-4/50005-9.

21 R. L. Puurunen, Appl. Surf. Sci., 2005, 245, 6-10. 
22 K. Sawai, R. Tatumi, T. Nakahodo and H. Fujihara, Angew. Chem., 2008, 120, 7023-7025.

23 G. M. Scheuermann, L. Rumi, P. Steurer, W. Bannwarth and R. Mülhaupt, J. Am. Chem. Soc., 2009, 131, 8262-8270.

24 C. C. Cassol, A. P. Umpierre, G. Machado, S. I. Wolke and J. Dupont, J. Am. Chem. Soc., 2005, 127, 3298-3299.

25 A. Corma, S. Iborra, F. X. Llabrés i Xamena, R. Montón, J. J. Calvino and C. Prestipino, J. Phys. Chem. C, 2010, 114, 8828-8836.

26 E. H. Rahim, F. S. Kamounah, J. Frederiksen and J. B. Christensen, Nano Lett., 2001, 1, 499-501.

27 D. B. Pacardo, M. Sethi, S. E. Jones, R. R. Naik and M. R. Knecht, ACS Nano, 2009, 3, 1288-1296.

28 J. C. Garcia-Martinez, R. Lezutekong and R. M. Crooks, J. Am. Chem. Soc., 2005, 127, 5097-5103.

29 S. U. Son, Y. Jang, J. Park, H. B. Na, H. M. Park, H. J. Yun, J. Lee and T. Hyeon, J. Am. Chem. Soc., 2004, 126, 5026-5027.

30 A. Elhage, A. E. Lanterna and J. C. Scaiano, ACS Sustainable Chem. Eng., 2018, 6, 1717-1722.

31 F. Topuz and T. Uyar, J. Colloid Interface Sci., 2019, 544, 206216.

32 C. Chen, Y. Tang, B. Vlahovic and F. Yan, Nanoscale Res. Lett., 2017, 12, 451.

33 S. Nishimura, Handbook of Heterogeneous Catalytic Hydrogenation for Organic Synthesis, Wiley, Chichester, 2001, pp. 315-387.

34 L. Marcela Silva, S. Camila Filizzola de Andrade, S. Bruno Leonardo, S. Cristina Maria de, R. Jonas Pereira, C. Geovanni Dantas, S.-F. Elaine Maria de, A. Ricardo Jose, O. Mônica Cristina de and O. Renata Barbosa de, AntiCancer Agents Med. Chem., 2015, 15, 206-216.

35 C. W. Cheung, M. L. Ploeger and X. Hu, Nat. Commun., 2017, 8, 14878.

36 L. A. Reinke and M. J. Moyer, Drug Metab. Dispos., 1985, 13, 548-552.
37 R. H. Hill, S. L. Head, S. Baker, M. Gregg, D. B. Shealy, S. L. Bailey, C. C. Williams, E. J. Sampson and L. L. Needham, Environ. Res., 1995, 71, 99-108.

38 Y. Zhao, L. Liu, D. Shi, X. Shi and M. Shen, Nanoscale Adv., 2019, 1, 342-346.

39 M. Orlandi, D. Brenna, R. Harms, S. Jost and M. Benaglia, Org. Process Res. Dev., 2018, 22, 430-445.

40 A. Celebioglu, Z. I. Yildiz and T. Uyar, Sci. Rep., 2017, 7, 7369.

41 A. Celebioglu, F. Topuz, Z. I. Yildiz and T. Uyar, ACS Omega, 2019, 4, 7850-7860.

42 A. Celebioglu, F. Topuz and T. Uyar, ACS Appl. Polym. Mater., 2019, 1, 54-62.

43 B. Martel, D. Ruffin, M. Weltrowski, Y. Lekchiri and M. Morcellet, J. Appl. Polym. Sci., 2005, 97, 433-442.

44 F. Grillo, H. Van Bui, J. A. Moulijn, M. T. Kreutzer and J. R. van Ommen, J. Phys. Chem. Lett., 2017, 8, 975-983.

45 J. Dendooven, R. K. Ramachandran, E. Solano, M. Kurttepeli, L. Geerts, G. Heremans, J. Rongé, M. M. Minjauw, T. Dobbelaere, K. Devloo-Casier, J. A. Martens, A. Vantomme, S. Bals, G. Portale, A. Coati and C. Detavernier, Nat. Commun., 2017, 8, 1074.

46 S. Navaladian, B. Viswanathan, T. K. Varadarajan and R. P. Viswanath, Nanoscale Res. Lett., 2008, 4, 181-186.

47 M. S. Dieckmann and K. A. Gray, Water Res., 1996, 30, 11691183.

48 N. Takahashi, T. Nakai, Y. Satoh and Y. Katoh, Water Res., 1994, 28, 1563-1570.

49 E. Lipczynska-Kochany, Environ. Technol., 1991, 12, 87-92.

50 K. S. Ranjith, A. Celebioglu and T. Uyar, Nanotechnology, 2018, 29, 245602.

51 Z.-R. Yang, S.-Q. Wang, J. Wang, A.-J. Zhou and C.-W. Xu, Sci. Rep., 2017, 7, 15479.

52 M. M. Demir, M. A. Gulgun, Y. Z. Menceloglu, B. Erman, S. S. Abramchuk, E. E. Makhaeva, A. R. Khokhlov, V. G. Matveeva and M. G. Sulman, Macromolecules, 2004, 37, 1787-1792. 